\title{
Resultados de la cirugía endoscópica de la glándula tiroides por vía transaxilar en Perú
}

\author{
Results of transaxillary endoscopic surgery of the thyroid gland in Peru \\ Alvaro Díaz ${ }^{1, a}$, Jonathan Díaz ${ }^{2, b}$, José Novoa ${ }^{3, b}$, Edward Bustamante ${ }^{3, b}$, Juan Postigo ${ }^{4, c}$ \\ RESUMEN
}

Objetivo: Describir los resultados de la tiroidectomía endoscópica transaxilar sin insuflación de $\mathrm{CO}_{2}$ en Perú. Material y métodos: Estudio descriptivo, retrospectivo de una serie de casos basado en la revisión de las historias clínicas de los pacientes con tumor tiroideo sometidos a tiroidectomía endoscópica transaxilar sin insuflación de $\mathrm{CO}_{2}$ entre noviembre 2014 y marzo 2017, en instituciones públicas y privadas del sistema nacional de salud de la ciudad de Lima. Resultados: Se realizaron 24 tiroidectomías endoscópicas, de las cuales 15 fueron hemitiroidectomia, 8 tiroidectomía total y una tiroidectomía subtotal. El tiempo operatorio promedio fue de 193,8 minutos. Las complicaciones más frecuentes fueron el hematoma postoperatorio $(12,5 \%)$ seguido de la hipocalcemia transitoria (8,3\%); ningún caso presentó lesión del plexo braquial. Conclusiones: La tiroidectomía endoscópica por vía transaxilar es un procedimiento seguro, factible de realizar en instituciones de salud que cuenten con el equipamiento de cirugía video endoscópica, que podría indicarse en determinados pacientes.

PALABRAS CLAVE: Tiroidectomía, procedimientos quirúrgicos mínimamente invasivos, cirugía asistida por video. (Fuente: DeCS BIREME).

\section{SUMMARY}

Objective: To describe the results of transaxillary endoscopic surgery of the thyroid gland without insufflating CO2 in Peru. Methods: A retrospective case series based of chart review of patients with thyroid cancer undergoing transaxillary endoscopic surgery of the thyroid gland without insufflating CO2 from November 2014 to March 2017 in public and private institutions in Lima. Results: 24 transaxillary endoscopic procedures were performed, 15 of which were hemithyroidectomies, 8 were total thyroidectomies and one subtotal thyroidectomy. Mean operating time was 193.8 minutes. The most common complication was post operatory hematoma $(12.5 \%)$ followed by transient hypocalcemia (8.3\%), no lesions of the brachial plexus were observed. Conclusions: Transaxillary endoscopic surgery of the thyroid gland is a safe and feasible procedure to be performed in equipped centers.

KEYWORDS: Thyroidectomy, minimally invasive surgical procedures, video-assisted surgery. (Source: MeSH NLM).

\footnotetext{
Departamento de Cirugía de Cabeza y Cuello, Instituto Nacional de Enfermedades Neoplásicas. Lima, Perú.

Servicio de Cirugía de Especialidades II, Hospital de Alta Complejidad Virgen de la Puerta. Lima, Perú.

Servicio de Cirugía de Cabeza, Cuello y Maxilofacial, Hospital Nacional Arzobispo Loayza. Lima, Perú.

Cirugía de Cabeza y Cuello, Clínica Oncosalud, AUNA. Lima, Perú.

Magister en medicina con mención en cirugía oncológica

Bachiller en medicina

Doctor en medicina
} 


\section{INTRODUCCIÓN}

La cirugía de la glándula tiroides tal como se conoce hoy en día establece sus fundamentos a finales del siglo XIX gracias a la valiosa contribución del Dr. Kocher al conocimiento de la fisiología y anatomía quirúrgica de este órgano, lo que permitió reducir la morbilidad y mortalidad postoperatoria de manera ostensible, por lo que es reconocido como el padre de la cirugía moderna.

Los avances tecnológicos de las últimas décadas y el desarrollo de la cirugía mínimamente invasiva en el tratamiento de diversas patologías no ha sido ajeno a la especialidad de cirugía de cabeza y cuello. En 1996, Hüscher (1), realizó la primera tiroidectomía video asistida y en el año 2000 los japoneses publicaron su experiencia en tiroidectomía endoscópica mediante accesos mamario o transaxilar con insuflación de $\mathrm{CO}_{2}$ (2). En Perú, Granda y Ortega realizaron hemitiroidectomía en 5 casos en el Hospital de EsSalud de Huancayo entre los años 1998 y 1999 mediante un acceso cervical endoscópico (3). Todas estas técnicas utilizaron insuflación de gas $\mathrm{CO}_{2}$ para generar el espacio de trabajo, replicando lo realizado en cirugía endoscópica abdominal; sin embargo, las características anatómicas de la región cervical determinan el colapso constante del área de trabajo que dificulta la visibilidad durante el procedimiento. Con la idea de superar este inconveniente, el grupo surcoreano liderado por Chung (4), desarrolló una variante de la tiroidectomía transaxilar endoscópica al prescindir de la utilización de gas, posteriormente incorporan la cirugía robótica y en la actualidad, ostentan la experiencia más grande del mundo en tiroidectomía por vía transaxilar sin insuflación de $\mathrm{CO}_{2}(5)$.

Para el año 2011 en pleno crecimiento del interés de la comunidad médica internacional en la cirugía mínimamente invasiva, no existían más de 13 robots en toda Latinoamérica, por lo que el desarrollo de esta técnica ha sido más tardío en esta parte del continente (6). Hoy en día, el alto costo de implementación y mantenimiento que representa, continua siendo una de las limitantes más importante para montar un centro de cirugía robótica, por lo que la aplicación de técnicas de cirugía no robótica permanecen vigentes en la mayoría de instituciones de salud que cuenten con equipamiento e instrumental para cirugía endoscópica en general, siendo factible ofrecer esta alternativa de tratamiento quirúrgico a determinados pacientes con tumores tiroideos en nuestra región.
La primera tiroidectomía endoscópica sin insuflación de gas por vía transaxilar en Perú fue realizada en el servicio de cirugía de cabeza y cuello del Hospital Nacional Arzobispo Loayza en el año 2014 (7).

El objetivo del estudio fue describir los resultados de la tiroidectomía endoscópica transaxilar sin insuflación de $\mathrm{CO} 2$ en Perú.

\section{MATERIAL Y MÉTODOS}

Estudio observacional, descriptivo, de corte transversal basado en la población de pacientes sometidos a tiroidectomía endoscópica transaxilar entre noviembre 2014 y marzo 2017 en diferentes instituciones de salud a nivel nacional.

Se incluyeron los pacientes sometidos a tiroidectomía endoscópica transaxilar que tuvieron seguimiento de al menos 24 meses y que contaran con información de sonoelastografía tiroidea preoperatoria, biopsia por aspiración con aguja fina, estudio de funcionalidad laríngea con nasolaringoscopía y estudio electrofisiológico del plexo braquial antes y después de la cirugía, así como, determinación de los niveles de paratohormona intacta (iPTH) y calcio iónico $\left(\mathrm{Ca}^{++}\right)$ en los pacientes sometidos a tiroidectomía total. La información se obtuvo de los registros médicos.

Para fines del análisis del tiempo operatorio se consideró el tiempo medido en minutos desde el inicio de la cirugía hasta que se completó la tiroidectomía

Tabla 1. Características demográficas y clínicas de los pacientes incluidos en el estudio $(n=24)$.

\begin{tabular}{lcr}
\hline Características & $\mathbf{n}$ & \% \\
\hline Edad & & \\
$<20$ & 2 & 8,4 \\
$21-30$ & 6 & 25,0 \\
$31-40$ & 5 & 20,8 \\
$41-50$ & 5 & 20,8 \\
$>51$ & 6 & 25,0 \\
$\mathrm{IMC}^{(*)}$ & & \\
$<25$ & 16 & 66,7 \\
$>25$ & 8 & 33,3 \\
Género & & \\
Femenino & 23 & 95,8 \\
Masculino & 1 & 4,1 \\
Total & 24 & 100,0 \\
\hline *IMC: Índice de mas corpol $\left(\mathrm{kg}^{2} \mathrm{~m}^{2}\right)$ &
\end{tabular}

*IMC: Índice de masa corporal $\left(\mathrm{kg} / \mathrm{m}^{2}\right)$ 
endoscópica. Así mismo, el tiempo fue analizado en función del número de minutos empleados en cada una de las tres etapas del procedimiento: generación del espacio de trabajo, colocación de retractores autoestáticos y, la tiroidectomía endoscópica propiamente dicha. Para analizar la curva de aprendizaje en función del tiempo operatorio se agruparon a los pacientes sometidos a tiroidectomía endoscópica en grupos de tres en orden cronológico sucesivo.

Se utilizó estadística descriptiva para la presentación de los datos.

El estudio fue exonerado de revisión por el Comité de ética por ser de carácter observacional.

\section{RESULTADOS}

Se realizaron 24 tiroidectomías entre noviembre 2014 y marzo 2017, 95\% de los casos correspondieron a pacientes de sexo femenino, el $80 \%$ tenían edades comprendidas entre los 30 y 50 años de edad, y el 66\% tenían un IMC menor a 25 (tabla 1).

El 45\% de los tumores de tiroides tenían un tamaño comprendido entre 20,1 y $40 \mathrm{~mm}$, la lateralidad del tumor fue equiparable. Todos los pacientes tuvieron estudios de biopsia por aspiración con aguja fina, de los cuales solo el 8,3\% de los casos tuvieron un resultado de citología insatisfactoria (tabla 2).

Tabla 2. Características clínicas y citología del tumor tiroideo en pacientes sometidos a tiroidectomía endoscópica transaxilar $(\mathrm{n}=24)$.

\begin{tabular}{lcr}
\hline Características & $\mathbf{n}$ & $\mathbf{\%}$ \\
\hline Tamaño & & \\
$<10 \mathrm{~mm}$ & 4 & 16,7 \\
$10.1-20 \mathrm{~mm}$ & 9 & 37,5 \\
$20.1-40 \mathrm{~mm}$ & 11 & 45,8 \\
Lateralidad & & \\
$\quad$ Derecho & 13 & 54,2 \\
Izquierdo & 11 & 45,8 \\
Citología (Sistema Bethesda) & & \\
I & 2 & 8,3 \\
II & 10 & 41,7 \\
III & 2 & 8,3 \\
IV & 9 & 37,5 \\
VI & 1 & 4,2 \\
Total & 24 & 100,0 \\
\hline
\end{tabular}

Los resultados del estudio anátomo-patológico se muestran en la tabla 3, los más frecuentes fueron bocio (hiperplasia multinodular, hiperplasia con degeneración quística) y carcinoma papilar.

Se realizó tiroidectomía total endoscópica en ocho pacientes, hemitiroidectomia endoscópica en 15 y solo en un paciente se realizó tiroidectomía subtotal endoscópica. La extensión de la cirugía en pacientes con diagnóstico de cáncer de tiroides fue tiroidectomía total endoscópica en el $50 \%$ de los casos, el resto tuvieron hemitiroidectomia o tiroidectomía subtotal endoscópica.

El tiempo operatorio global promedio fue de 193,8 minutos (rango de 100 a 310 minutos). El tiempo operatorio fue de 181,5 minutos (rango de 100 a 255 minutos) para la hemitiroidectomia endoscópica, y de 214,3 minutos (rango de 130 a 310 minutos) para la tiroidectomía total y subtotal endoscópica.

El tiempo empleado en la primera etapa del procedimiento, es decir, la generación del espacio de trabajo fue 77,6 minutos (rango de 45 a 200 minutos), en la colocación de retractores autoestáticos fue 11,4 minutos (rango 5 a 25 minutos), y en la tiroidectomía endoscópica propiamente dicha fue 103,6 minutos (rango de 35 a 240 minutos).

Tabla 3. Diagnóstico patológico del tumor tiroideo $(\mathrm{n}=24)$.

\begin{tabular}{lcc}
\hline Diagnóstico patológico & $\mathbf{n}$ & $\mathbf{\%}$ \\
\hline Hiperplasia nodular & 3 & 12,5 \\
Carcinoma papilar & 6 & 25,0 \\
Bocio & 10 & 41,6 \\
Adenoma folicular & 4 & 16,7 \\
Tiroiditis & 1 & 4,2 \\
Total & 24 & 100,0 \\
\hline
\end{tabular}

Tabla 4. Complicaciones en tiroidectomía endoscópica transaxilar.

\begin{tabular}{lcr}
\hline Complicaciones & n (\%) & \multicolumn{1}{c}{$\%$} \\
\hline Disfonía transitoria & $1(4)$ & 4,0 \\
Lesión del nervio recurrente & $1(4)$ & 4,0 \\
Hipocalcemia transitoria & $2(8)$ & 8,0 \\
Hematoma postoperatorio & $3(12)$ & 12,0 \\
Dolor de hombro & $1(4)$ & 4,0 \\
\hline
\end{tabular}


El tiempo operatorio global en la hemitiroidectomía endoscópica fue 85 minutos (rango 35 a 180 minutos) y 135,8 minutos (rango 75 a 240 minutos) en la tiroidectomía total endoscópica incluyendo la subtotal: HT y TT TO (gráfico 1 y gráfico 2).

El tiempo quirúrgico total fue decreciendo principalmente en función de la reducción del tiempo empleado en el primer paso de la cirugía, es decir, la disección del colgajo axilar y generación del espacio de trabajo (gráfico 3).

En la tabla 4, se pueden observar las complicaciones, las más frecuentes fueron el hematoma postoperatorio seguido por la hipocalcemia transitoria, en tres $(12,5 \%)$ y dos $(8,3 \%)$ casos, respectivamente. Se presentó un caso de lesión permanente de nervio laríngeo recurrente en un paciente sometido a hemitiroidectomia. No se presentaron casos de lesión del plexo braquial demostrado por los estudios electrofisiológicos. La estancia hospitalaria promedio fue de 2,12 $\pm 0,34$ días.

El seguimiento global de estos pacientes fue de 24 a 52 meses. En el caso de los pacientes con el diagnóstico patológico de cáncer de tiroides el seguimiento fue de 24 a 49 meses, no evidenciando recurrencia de enfermedad hasta su último control.

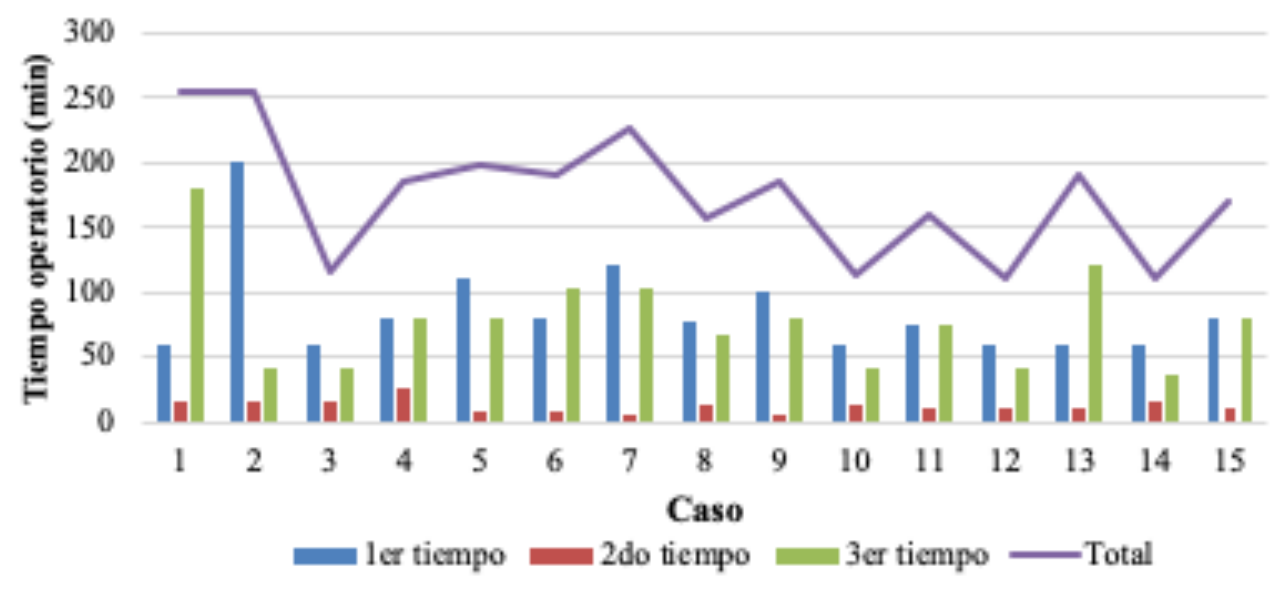

Gráfico 1. Tiempo operatorio en hemitiroidectomía endoscópica transaxilar.

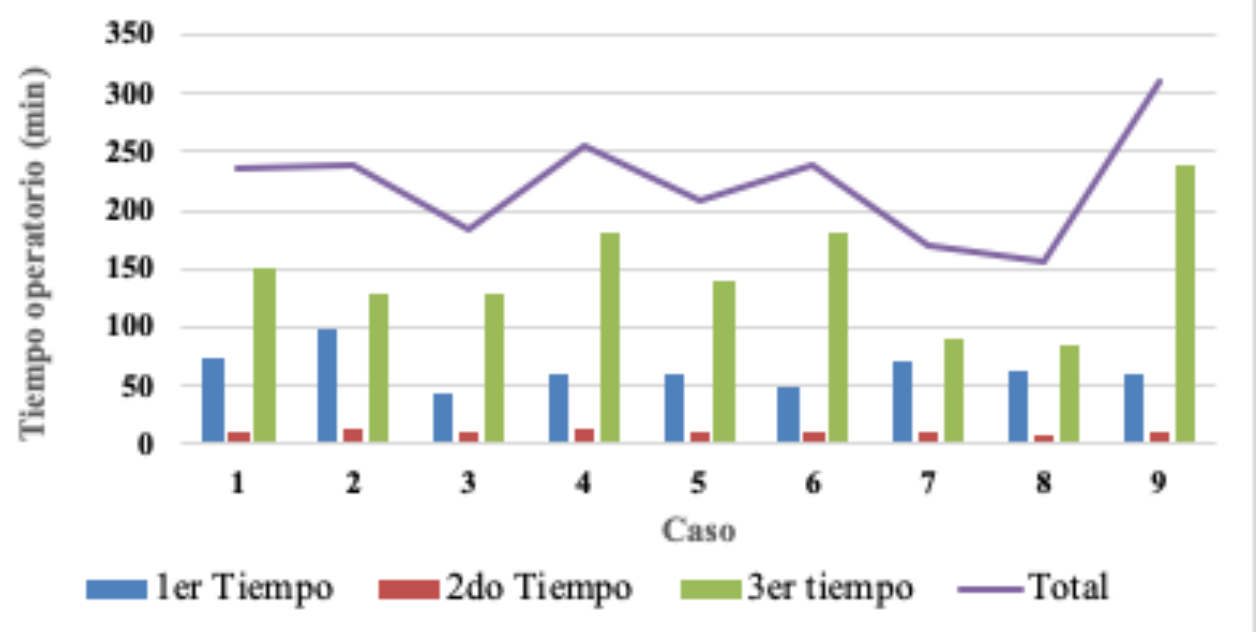

Gráfico 2. Tiempo operatorio en tiroidectomía total/subtotal endoscópica transaxilar. 


\section{INVESTIGACIÓN ORIGINAL I ORIGINAL RESEARCH}

Díaz. A. y col.

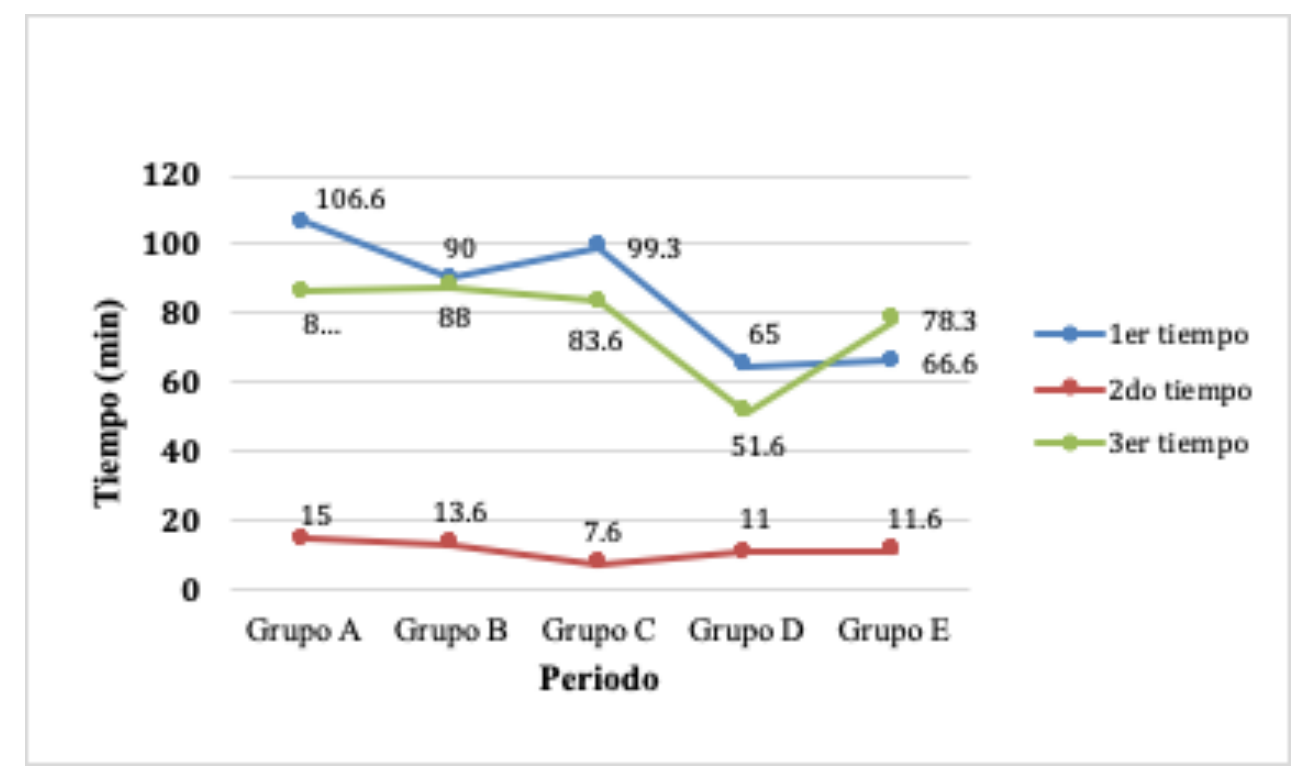

Gráfico 3. Curva de aprendizaje. Tiempo operatorio en hemitiroidectomía endoscópica transaxilar $(\mathrm{n}=15)$.

\section{DISCUSIÓN}

La tiroidectomía endoscópica puede clasificarse en técnicas directas e indirectas también llamadas de acceso remoto, siendo las primeras las que se realizan mediante la introducción de los instrumentos directamente en la región cervical. Las técnicas indirectas comprenden los abordajes remotos como el retroauricular, mamario y axilar, que a su vez pueden subdividirse en razón al uso o no de insuflación de gas $\mathrm{CO}_{2}$, o según la tecnología empleada como cirugía robótica o cirugía endoscópica propiamente dicha.

El abordaje transaxilar fue desarrollado en el año 2000 por Ikeda (8), y modificado por Chung para no utilizar la insuflación de gas CO2 (9), evitando el colapso del área de trabajo durante la cirugía. En comparación con otros accesos remotos, la tiroidectomía endoscópica transaxilar permite manejar el lóbulo tiroideo contralateral por el mismo abordaje y completar la tiroidectomía total (10).

Ciertamente, existen algunas consideraciones importantes a tomar en cuenta al momento de la selección del caso, que pueden clasificarse en factores dependientes del tumor y otros inherentes al paciente. En general, el nódulo debe ser menor a $3 \mathrm{~cm}$ y el lóbulo tiroideo no mayor a $5 \mathrm{~cm}$, no tener evidencia de tiroiditis asociada, extensión retroesternal o metástasis ganglionar. Por otro lado, en cuanto a los factores dependientes del paciente este debe tener idealmente un índice de masa corporal (IMC) no mayor a 30, una distancia entre la línea axilar anterior y la lineo medio cervical de hasta $17 \mathrm{~cm}, \mathrm{y}$ no tener antecedente de cirugía previa o radioterapia en el cuello (10).

La serie más grande en el mundo en tiroidectomia transaxilar sin insuflación de gas la tiene el grupo de Corea del Sur con 5000 casos (11). El tiempo operatorio global fue de 134 minutos (rango 53 - 635 minutos); $113,18 \pm 28,6$ y $141,98 \pm 34,1$ minutos para las hemitiroidectomias y tiroidectomias totales, respectivamente. La conformación del colgajo axilar tomó de 37,9 $\pm 13,9$ minutos, colocación de retractores 4,64 $\pm 2,2$ minutos, y la tiroidectomía propiamente dicha, 41,65 $\pm 17,65$ minutos en las hemitiroidectomia y $63,4 \pm 21,5$ minutos en la tiroidectomía total.

La estancia hospitalaria fue de 3,3 \pm 3 días (rango 2-7). La disfonía transitoria y el seroma fueron las complicaciones más frecuentes, alcanzando el 2,5\% y $1,15 \%$ de los casos. En el caso de las tirodectomias totales se observó hipoparatiroidismo transitorio en el $48,1 \%$ de los casos, siendo permanente en el 1,3\%.

La experiencia en Estados Unidos de Norteamérica fue algo distinta, el $60 \%$ de sus pacientes eran obesos, lo que determinó mayor tiempo operatorio y número de complicaciones, lo que los impulsó a desarrollar otros abordajes como el retroauricular, que ha sido adoptado en Brasil desde el 2014, convirtiéndose este país en el pionero del uso de la técnica retroauricular en esta parte del continente, coincidiendo con el nacimiento de la tiroidectomía endoscópica transaxilar sin insuflación de gas $\mathrm{CO} 2$ en el Perú en el mismo año $(7,12)$. 
El tiempo operatorio promedio en nuestra serie fue de 193,8 minutos, comparado con 126,8 y 131 minutos en las series iniciales de Corea del Sur y Estados Unidos de Norteamérica, respectivamente. Cabe mencionar, que las series coreana y estadounidense solo comprendieron cirugía parcial de la glándula tiroides como hemitiroidectomia (lobectomías y hemitiroidectomias propiamente dichas), en cambio en nuestra serie se incluyó tanto cirugías parciales como totales, lo que explica en parte el mayor tiempo operatorio. La estancia hospitalaria promedio fue de 4 días en el estudio coreano y de un día en el estudio norteamericano $(4,13)$, en nuestro caso fue 2 días.

El impacto de la curva de aprendizaje en la reducción de los tiempos operatorios puede verse reflejado en el análisis de las curvas de tiempo para las hemitiroidectomias en la presente serie, principalmente a expensas de la reducción en el tiempo empleado en la preparación del colgajo axilar, lo que refleja el efecto de la experiencia acumulada progresivamente. La curva de aprendizaje para esta técnica involucra también a todo el equipo humano en sala de operaciones, lo que explica el incremento en el tiempo operatorio en los últimos casos de la presente serie, a expensas del tercer tiempo quirúrgico de esta técnica, que correspondieron al momento en que se inició la implementación de la tiroidectomía endoscópica en otros centros quirúrgicos no habituados a este acceso.

No se han encontrado diferencia significativa en las complicaciones en cirugía convencional y tiroidectomía endoscópica (14); sin embargo, se reportan ventajas como no alteración sensorial de la región cervical anterior, menor dificultad para la deglución, y mayor nivel de satisfacción con el resultado cosmético (15). Las complicaciones descritas en nuestra serie no difieren de las reportadas en la literatura mundial para tiroidectomía convencional, lo que demuestra la seguridad de la técnica transaxilar.

Algunas de las experiencias internacionales no fueron muy afortunadas respecto a la lesión del plexo braquial, que se explica por las características del paciente con IMC elevado y por la hiperextensión del brazo como se realizaba inicialmente en los primeros años de la aplicación de esta técnica, lo que se evita actualmente con una adecuada selección del paciente y evitando la sobrecarga del plexo, al flexionar el antebrazo sobre el nivel de la cabeza un poco por encima del nivel de la frente, en una posición cómoda previamente valorada con el paciente despierto antes de la inducción de la anestesia.
El $25 \%$ de los casos en la presente serie correspondieron a pacientes con cáncer de tiroides, siendo tratados con hemitiroidectomía endoscópica la mitad de ellos, lo que se ajusta a las recomendaciones de la Asociación Americana de Tiroides respecto a la extensión de la tiroidectomia para el tratamiento del cáncer de tiroides (16), no se ha demostrado diferencia en sobrevida cuando se comparan los pacientes sometidos a hemitiroidectomia versus aquellos con tiroidectomía total $(17,18)$. En nuestra serie no se evidenció recurrencia de enfermedad durante el tiempo de seguimiento.

La implementación de nuevas técnicas quirúrgicas, que demandan muchas veces recursos tecnológicos de alto costo, encuentran fuertes dificultades para desarrollarse en un sistema de salud con presupuesto limitado como el nuestro. En América Latina para el 2016 existían 53 sistemas robóticos, de los cuales casi el 50\% se encontraban en Brasil (19), lo que explica su mayor casuística en comparación con el Perú, que a la fecha no cuenta con ningún robot. La otra dificultad a la que se enfrentan los cirujanos que adoptan estas técnicas novedosas, es lidiar con los paradigmas existentes para vencer la resistencia natural de algunos colegas habituados a los procedimientos quirúrgicos convencionales, por lo que se requiere trabajar de manera sistematizada y con rigor científico a fin de demostrar las ventajas y aplicaciones de estas técnicas.

Debido al reducido número de casos de la presente serie y la falta de grupo comparativo no es posible establecer conclusiones válidas para recomendar este procedimiento.

En conclusión, la tiroidectomía endoscópica por vía transaxilar constituye el acceso remoto más versátil para poder realizar los procedimientos de hemitiroidectomia y tiroidectomía total por un solo acceso, con los recursos tecnológicos existentes en la mayoría de instituciones de salud que cuenten con equipos de videoendoscopía. Es una procedimiento seguro que tiene indicaciones precisas para determinado grupo de pacientes, las complicaciones descritas son las mismas que las contempladas en la tiroidectomía convencional.

\section{Declaración de financiamiento y de conflictos de interés:}

El estudio fue financiado por los autores. Los autores declaran no tener conflictos de interés. 


\section{Contribución de autoría:}

AD: Recolección y análisis de la data, análisis y redacción del artículo; JD, JN, y EB: Recolección y análisis de los datos; JP: Revisión del artículo.

\section{Correspondencia:}

Alvaro Díaz Cárdenas

Instituto Nacional de Enfermedades Neoplásicas

Avenida Angamos Oeste 2560 Oficina 214, Surquillo. Lima, Perú.

Correo electrónico: alvaro.diaz.cardenas@upch.pe

\section{REFERENCIAS BIBLIOGRÁFICAS}

1. Hüscher CS, Chiodini S, Napolitano C, et al. Endoscopic right thyroid lobectomy. Surg Endosc. 1997; 11:877.

2. Ikeda Y, Takami H, Niimi M, et al. Endoscopic thyroidectomy by the axillary approach. Surg Endosc. 2001; 15:1362-1364.

3. Granda LA, Ortega F. Tiroidectomia endoscópica: nueva técnica para el manejo del nódulo benigno de la glandula tiroides. Huancayo: Hospital Nacional Huancayo Essalud; 2007. (Citado el 25 de mayo del 2019), Disponible en: www.geocities. ws/jpardalr/tirendosc.pdf

4. Yoon JH, Park CH, Chung WY. Gasless endoscopic thyroidectomy via an axillary approach: Experience of 30 cases. Surg Laparosc Endosc Percutan Tech. 2006; 16:226-231.

5. Kim MJ, Nam KH, Lee SG, et al. Yonsei experience of 5000 gasless transaxillary robotic thyroidectomies. World J Surg. 2018; 42(2):393401.

6. Vaessen C. Location of robotic surgical systems worldwide and in France. J Visc Surg. 2011; 148(5):e9-11.

7. Díaz A. Tiroidectomía endoscópica por vía transaxilar (Para un tumor benigno de la tiroides). Diagnóstico. 2015; 54(3):153-56.

8. Ikeda Y, Takami H, Sasaki Y, Kan S, Niimi M. Endoscopic resection of thyroid tumors by the axillary approach. J Cardiovasc Surg (Torino). 2000; 41:791-792.

9. Yoon JH, Park CH, Chung WY. Gasless endoscopic thyroidectomy via an axillary approach: experience of 30 cases. Surg Laparosc Endosc Percutan Tech. 2006; 16:226-231.
10. Berber E, Bernet V, Fahey TJ 3rd, et al. American Thyroid Association Statement on Remote - Access Thyroid Surgery. Thyroid. 2016; 26(3):331-337.

11. Kim MJ, Nam KH, Lee SG, et al. Yonsei Experience of 5000 Gasless Transaxillary Robotic Thyroidectomies. World J Surg. 2018; 42(2):393401.

12. Terris DJ, Singer MC, Seybt MW. Robotic facelift thyroidectomy: II. Clinical feasibility and safety. Laryngoscope. 2011; 121(8):1636-1641.

13. Kandil E, Winters R, Aslam R, Friedlander P, Bellows C. Transaxillary gasless robotic thyroid surgery with nerve monitoring: initial two experience in a North American center. Minim Invasive Ther Allied Technol. 2012; 21(2):90-95.

14. Hakim Darail NA, Lee SH, Kang SW, Jeong JJ, Nam KH, Chung WY. Gasless transaxillary endoscopic thyroidectomy: a decade on. Surg Laparosc Endosc Percutan Tech. 2014; 24(6):e211215.

15. Lee J, Kwon IS, Bae EH, Chung WY. Comparative analysis of oncological outcomes and quality of life after robotic versus conventional open thyroidectomy with modified radical neck dissection in patients with papillary thyroid carcinoma and lateral neck node metastases. J Clin Endocrinol Metab. 2013; 98:2701-2708.

16. Haugen BR, Alexander EK, Bible KC, et al. 2015 American Thyroid Association Management Guidelines for Adult Patients with Thyroid Nodules and Differentiated Thyroid Cancer: The American Thyroid Association Guidelines Task Force on Thyroid Nodules and Differentiated Thyroid Cancer. Thyroid. 2016; 26(1):1-133.

17. Barney BM, Hitchcock YJ, Sharma P, Shrieve DC, Tward JD. Overall and cause-specific survival for patients undergoing lobectomy, near-total, or total thyroidectomy for differentiated thyroid cancer. Head Neck. 2011; 33:645-649.

18. Mendelsohn AH, Elashoff DA, Abemayor E, St John MA. Surgery for papillary thyroid carcinoma: is lobectomy enough? Arch Otolaryngol Head Neck Surg. 2010; 136:1055-1061.

19. Lira RB, Chulam TC, Kowalski LP. Safe implementation of retroauricular robotic and endoscopic neck surgery in South America. Gland Surg. 2017; 6(3):258-266.

Recibido: $19 / 06 / 2019$

Aceptado: 24/09/2020 